\title{
Plasma wake inhibition at the collision of two laser pulses in an underdense plasma
}

\author{
C. Rechatin, ${ }^{1}$ J. Faure, ${ }^{1}$ A. Lifschitz,${ }^{1}$ V. Malka,${ }^{1}$ and E. Lefebvre ${ }^{2}$ \\ ${ }^{1}$ Laboratoire d'Optique Appliquée, ENSTA, CNRS, \\ Ecole Polytechnique, UMR 7639, 91761 Palaiseau, France \\ ${ }^{2}$ Département de Physique Théorique et Appliquée, CEA, \\ DAM Ile-de-France, BP 12, 91680 Bruyères-le-Châtel, France
}

(Dated: October 18, 2018)

\begin{abstract}
An electron injector concept for laser-plasma accelerator was developed in ref [1] and [2] ; it relies on the use of counter-propagating ultrashort laser pulses. In [2], the scheme is as follows: the pump laser pulse generates a large amplitude laser wakefield (plasma wave). The counterpropagating injection pulse interferes with the pump laser pulse to generate a beatwave pattern. The ponderomotive force of the beatwave is able to inject plasma electrons into the wakefield. We have studied this injection scheme using 1D Particle in Cell (PIC) simulations. The simulations reveal phenomena and important physical processes that were not taken into account in previous models. In particular, at the collision of the laser pulses, most plasma electrons are trapped in the beatwave pattern and cannot contribute to the collective oscillation supporting the plasma wave. At this point, the fluid approximation fails and the plasma wake is strongly inhibited. Consequently, the injected charge is reduced by one order of magnitude compared to the predictions from previous models.
\end{abstract}

An intense laser pulse can drive an electrostatic plasma wave via the ponderomotive force which scales as $\nabla \mathbf{a}^{2}$, where $\mathbf{a}$ is the normalized potential vector of the laser $:|\mathbf{a}|=a=8.6 \times 10^{-10} \lambda[\mu \mathrm{m}] I_{0}^{1 / 2}\left[\mathrm{~W} . \mathrm{cm}^{-2}\right]$ for a linearly polarized laser. When the pulse duration is close to the plasma period $\left(\lambda_{p} / c\right)$, the laser pulse ponderomotive force pushes electrons and efficiently creates charge separation (ions hardly move). It results in a travelling longitudinal wave whose phase velocity $v_{p}$ is equal to the group velocity of the laser. In an underdense plasma, $v_{p}$ is very close to $c$, the speed of light, thus enabling acceleration of electrons to very high energies once they are trapped in the wake [3]. But in a linear or moderately nonlinear regime, an electron with no initial velocity is not trapped by this travelling wave and consequently, not accelerated. In a more nonlinear regime, transverse wave breaking effects can result in the self-trapping of electrons in the so-called "bubble regime" 4]. This phenomenon has been observed in 2004 in [5, 6, 7] where quasi monoenergetic electron beams at the $100 \mathrm{MeV}$ level were obtained. Nevertheless, in this scheme, self-injection and acceleration depend on the precise evolution of the laser pulse. Therefore, a fine control over the output electron beam is hard to achieve. On the contrary, precise control of electron injection would translate into good tailoring of the electron beam parameters, and would be most useful for applications [8, 9$]$.

To trap electrons in a regime where self-trapping does not occur, one has to externally inject electrons in the plasma wave, i.e. give electrons an initial momentum. In addition, electrons should be injected in a short time $\left(<\lambda_{p} / c\right)$ in order to produce a monoenergetic beam. This can be achieved using additional ultrashort laser pulses whose purpose is only restricted to triggering electron injection. Umstadter et al. [10] first proposed to use a second laser pulse propagating perpendicular to the pump laser pulse. The idea was to use the radial pon- deromotive kick of the second pulse to inject electrons. Esarey et al. 1] proposed a counter-propagating geometry based on the use of three laser pulses. This idea was further developed in Ref. [2], where only two laser pulses are necessary. In this scheme, a main pulse (pump pulse) with maximum amplitude $a_{0}$ creates a high amplitude plasma wave and collides with a secondary pulse of lower maximum amplitude $a_{1}$. The interference of the two beams creates a ponderomotive beatwave pattern with phase velocity $v_{b w}=0$, and thus enables to preaccelerate background electrons. Upon interacting with this field pattern, some background electrons gain enough momentum to be trapped in the main plasma wave and then accelerated to high energies. The force associated with this ponderomotive beatwave scales as $F_{b w}=2 k_{0} a_{0} a_{1}$, where $k_{0}$ is the central wavenumber of both pulses, $F_{b w}$ is many times greater than the ponderomotive force associated with the pump laser $F_{\text {pond }} \approx k_{p} a_{0}^{2}$ since in an underdense plasma $k_{0}>>k_{p}$. Therefore, the mechanism is still efficient even for modest values of $a_{0}$ and $a_{1}$. As the overlapping of the lasers is short in time, the electrons are injected in a very short distance and can be accelerated to an almost monoenergetic beam. This concept has been recently validated in an experiment [11], using two counter-propagating pulses. Each pulse had a duration of $30 \mathrm{fs}$ at full width half maximum (FWHM), with $a_{0}=1.3, a_{1}=0.4$. They were propagated in a plasma with electron density $n_{e}=7 \times 10^{18} \mathrm{~cm}^{-3}$ corresponding to $\gamma_{p}=k_{0} / k_{p}=15$. It was shown that the collision of the two lasers could lead to the generation of stable quasi-monoenergetic electron beams. The beam energy could be tuned by changing the collision position in the plasma.

The precise understanding of these experiments, as well as the optimization of this process, motivate the present study. We have used 1D Particle in cell (PIC) simulations to model electron injection in the plasma 
wave at the collision of the two lasers, and their subsequent acceleration. The PIC simulations are compared to existing models [1] and show major differences, such as the plasma fields behavior and the amount of injected charge.

We first describe the fluid model developped in Ref. [1, 2]. In the linear approximation the wakefield potential (due to charge separation) is a superposition of three potentials [12] : $\Phi=\Phi_{0}+\Phi_{1}+\Phi_{b} . \Phi_{0,1}$ are the charge separation potentials driven by the laser pulses $\mathbf{a}_{\mathbf{0}, \mathbf{1}}, \Phi_{0}$ representing the main accelerating structure we want to inject electrons in, and $\Phi_{b}$ is driven by the beatwave. The normalized expressions of the wakefields $\phi_{i}=e \Phi / m c$ are given by :

$$
\begin{array}{r}
\left(\frac{\partial^{2}}{\partial \xi_{0,1}^{2}}+k_{p}^{2}\right) \phi_{0,1}=\frac{k_{p}^{2}}{2}<\mathbf{a}_{\mathbf{0}, \mathbf{1}}{ }^{2}> \\
\left(\frac{\partial^{2}}{\partial t^{2}}+\omega_{p}^{2}\right) \phi_{b}=\omega_{p}^{2}<\mathbf{a}_{\mathbf{0}} \cdot \mathbf{a}_{\mathbf{1}}>
\end{array}
$$

where $\xi_{0,1}=z \mp v_{p} t$ stands for the phase relative to the pump and injection lasers, $k_{p}$ is the plasma wavevector and brackets $\langle$. $\rangle$ denote the time-average over the fast varying scale $\left(1 /\left(k_{0} c\right)\right)$. The term $<\mathbf{a}_{\mathbf{0}} \cdot \mathbf{a}_{\mathbf{1}}>$ is the beatwave, appearing only during the collision of the two lasers. Its spatial scale is given by $2 k_{0}$, for example for circularly polarized lasers we have $\mathbf{a}_{\mathbf{0}} \cdot \mathbf{a}_{\mathbf{1}}=$ $a_{0}\left(r, \xi_{0}\right) a_{1}\left(r, \xi_{1}\right) \cos \left(2 k_{0} z\right)$.

The first approximation of this analytical model consists of neglecting the last term, $\phi_{b}$. This is supported by the fact that the density variation linked with this electrostatic potential scales as $\delta n_{b} / n=4 k_{0}^{2} / k_{p}^{2} \phi_{b}$ with $k_{0} / k_{p}>>1$. This density variation being limited to $\delta n_{b} / n \simeq 1$ even in a non-linear regime, the potential can not be greater than $\phi_{b} \simeq k_{p}^{2} / 4 k_{0}^{2}$ which is often negligible compared to the other terms scaling as $\phi_{0,1} \simeq a_{0,1}^{2}$.

The second assumption is that we can separate the dynamics of the particles inside and outside the beatwave because the timescales are different (i.e. an electron will see a constant wakefield during an oscillation in a beatwave bucket). An underlying hypothesis here is that there are two different species of electrons, those maintaining the wakefield, or fluid electrons, and those being trapped in the beatwave, or test electrons.

Starting from these hypothesis, one can even build an analytical model when the lasers have modest intensities $\left(a_{0}<1\right)$ and are circularly polarized. In that case, the beatwave pattern is time independent. Using this analytical model, one can find an analytical threshold for trapping $([1,2]): 2 \sqrt{a_{0} a_{1}} \geq u_{z}\left(\xi_{0 \text { min }}\right)$ where $u_{z}\left(\xi_{0 \text { min }}\right)$ is the minimum normalized longitudinal momentum $p_{z} / m c$ for which the electrons can be trapped in the wakefield. The experiments described in ref[11] operated well above this threshold.

A numerical implementation of this model is a particle tracking code [2] where test particles are pushed in prescribed fields. The plasma fields are given by $\mathbf{E}_{\mathbf{p l}}=-\nabla\left(\Phi_{0}+\Phi_{1}\right)$ and the laser fields are solutions of the paraxial wave equation with a linear plasma response (gaussian beams). As expected, this model shows good agreement with the analytical model in the circular polarization case and allows to extend the scheme to the linear polarization case for which analytical theory is untractable. For linear polarization, the beatwave has a fast varying time dependence, which leads to stochastic effects [13]. In that case, the trapping thresholds are even lower.

The main results of this model, that we will later on refer to as the "prescribed fields" model, are that electrons can be injected with modest values of $a_{0}$ and $a_{1}$ (linear regime). The resulting bunch is quasi-monoenergetic because all the electrons are injected in a short distance (where the beating occurs). Moreover, the charge is expected to be up to some hundreds of $\mathrm{pC}$ in the linear regime. 1D PIC simulations, where fields are linked to the motion of electrons, have also already been carried out [14]. They confirmed that an electron beam with low energy spread, low emittance and short bunch length can be obtained with modest values of $a_{0}$ and $a_{1}$.

Here we compare 1D PIC simulations with a 1D prescribed field model. In this later model, we have used the 1D nonlinear theory of wakefield generation to be able to compare the results in a more nonlinear regime $\left(1<a_{0}<2\right)$. The corresponding equation writes $[15]$ :

$$
\frac{\partial^{2} \phi_{0,1}}{\partial \xi_{0,1}^{2}}=k_{p}^{2} \gamma_{p}^{2}\left[\beta_{p}\left(1-\frac{\left(1+<\mathbf{a}_{0,1}{ }^{2}>\right)}{\gamma_{p}^{2}\left(1+\phi_{0,1}\right)^{2}}\right)^{-1 / 2}-1\right]
$$

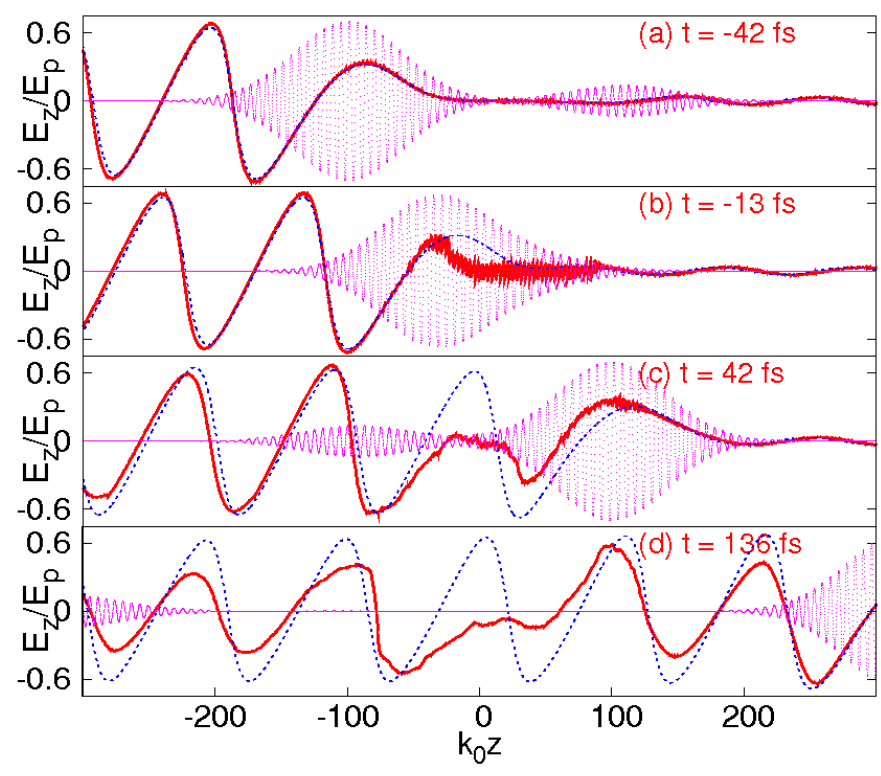

FIG. 1: Longitudinal electric field computed at different times in PIC simulation (thick solid line), and in prescribed fields simulation (dotted line).

1D PIC simulations were carried out with the code CALDER [16]. For all the simulation results presented here, we have used a simulation box of 6400 cells, each cell 
measuring $0.1 / k_{0}$. Both lasers were gaussian pulses with $30 \mathrm{fs}$ duration at FWHM and wavelength $\lambda_{0}=0.8 \mu \mathrm{m}$. To avoid edge effects, the main pulse entered the plasma through a density ramp of $100 \mu \mathrm{m}$, the maximum density being $7 \times 10^{18} \mathrm{~cm}^{-3}$. The simulation box was kept fixed to let the laser pulses enter the simulation correctly and we otherwise used a moving window to follow the main pulse over long propagation distances..

Fig. 1 shows the time evolution of the longitudinal electric field in 1D PIC simulations when the polarizations of the two pulses are parallel (solid line), compared to the fields externally specified by equation (3) (dotted line), for $a_{0}=2$ and $a_{1}=0.4$. The electric field is given in linear wave breaking field unit $E_{p}=c m_{e} \omega_{p} / e$ corresponding here to $E_{p}=250 \mathrm{GV} / \mathrm{m}$. The pump pulse propagates from left to right. We have also plotted the transverse electric field (thin dotted line) to show the position of the laser pulses. The collision occurs at $z=0$ at time $t=0$. Fig 1 a shows the electric field $40 \mathrm{fs}$ before the collision, it fits very well with the solution of equation (3). During the collision of the two beams (Fig,1.b) we clearly see a small spatial scale pattern at $2 k_{0}$, created by the beatwave. However, in this non-linear regime $\left(\delta n_{b} / n \simeq 1\right)$ we do not observe a superposition of this pattern with the usual wakefields as is the case in ref [12]. The most remarkable feature is the strong distorsion of the plasma wave at the position where the two pulses collide. This distorsion remains after a long time (Fig,1]c, 1,d) and a numerical estimate shows that the plasma wave amplitude is decreased by a factor of 10 at this position.
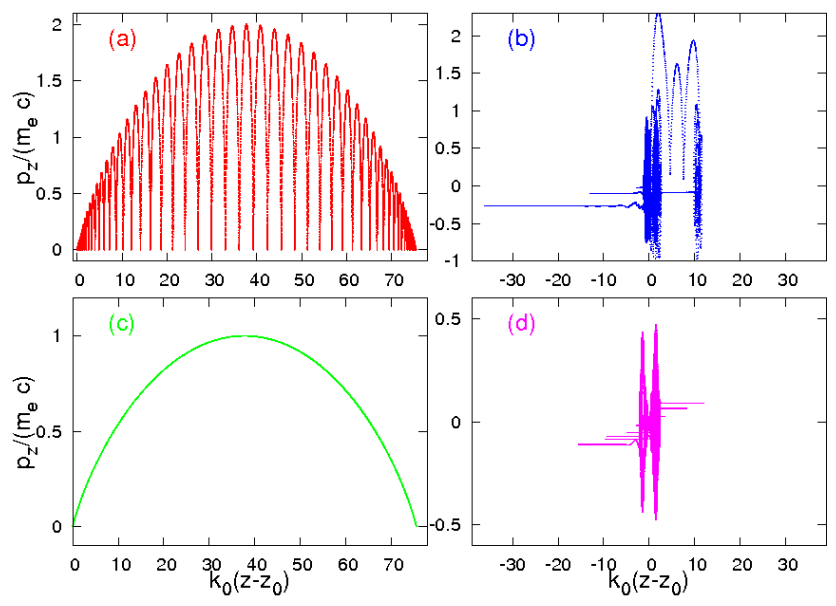

FIG. 2: Typical trajectories of electrons in phase space. a): Pump laser only, linear polarization $\left(a_{0}=2\right)$; b): Pump and injection lasers, parallel linear polarizations $\left(a_{0}=2\right.$ and $a_{1}=$ 0.4). c): Pump laser only, circular polarization $\left(a_{0}=2 / \sqrt{2}\right)$; d): Pump and injection lasers, circular polarizations $\left(a_{0}=\right.$ $2 / \sqrt{2}$ and $a_{1}=0.4 / \sqrt{2}$ )

This decrease of the wakefield amplitude follows from the fact that the electrons experiencing the collision of the lasers are trapped in the beatwave buckets and hence do not respond coherently (fluid-like) to the ponderomo- tive force of the main pulse. Without this fluid hypothesis, equation (3) fails. It would still hold if only some electrons were trapped, but here, as the phase velocity of the beatwave is zero, all electrons are trapped and kinetic effects cannot be treated as a perturbation.

To better understand this plasma wave "damping", we have performed simulations where test electrons are submitted to the laser fields in vacuum. In particular, this allows to understand electron motion in the ponderomotive beatwave. Results of these simulations are plotted in figure 2 where we represent the orbits of test electrons in $\left(z-z_{0}, p_{z}\right)$ phase space. Electrons are initially taken at rest, their initial position $z_{0}$ is randomly distributed around the collision position. Fig. 2, a illustrates the trajectories of electrons experiencing the pump laser field only (with $a_{0}=2$ ) : in the laser reference frame, every electron has the same motion because they are pushed by the same ponderomotive force. This fluid motion leads to the charge separation which drives the electrostatic field known as the plasma wave. On the contrary, when a parallel polarized injection beam is included, trajectories are drastically modified (Fig 2,b). Electrons are locally trapped in beatwave buckets, there is no large scale charge separation and hence the plasma wake is no longer excited. This wake inhibition phenomenon also occurs for circularly polarized lasers as shown in Figs 2 c and 2] d. This demonstrates that the plasma wave distorsion is not related to the stochastic motion of electrons, but really to their trapping in the beatwave buckets.

We now investigate the consequences of this plasma wake inhibition on the features of the electron beam. Figure 3 shows the energy of the electron beam versus their phase with respect to the laser (main plots) as well as the corresponding spectra (inserts) $300 \mu \mathrm{m}$ after the collision of the two beams. Fig 3 a corresponds to the prescribed field simulation and Fig 3 , b corresponds to the 1D PIC simulation. Qualitative differences on the energy spectra are only minor, both spectra showing a peaked distribution around $45 \mathrm{MeV}$. Electrons are trapped mainly in the first wakefield bucket, and less than ten percent are trapped in the next ones. However, depending on the model, the trapped charge differs by almost one order of magnitude.

In the prescribed fields model, electrons are preaccelerated in the beatwave. As their initial velocity is lower than the wakefield phase velocity, they slip backward in phase. They are then trapped in the wakefield provided the energy gained in the beatwave was sufficient. In that case, electrons catch up with the plasma wave and are accelerated to high energies. In the more realistic PIC simulation, the beatwave preaccelerates electrons in the same way but the wakefield is inhibited and most electrons just slip back in phase space without being trapped. In order to be trapped, electrons need to be preaccelerated at the end of the collision. As they slip back slower in phase space than fluid electrons, they witness a restored wakefield suitable for trapping and acceleration when they reach the back of the pump pulse. Therefore, 


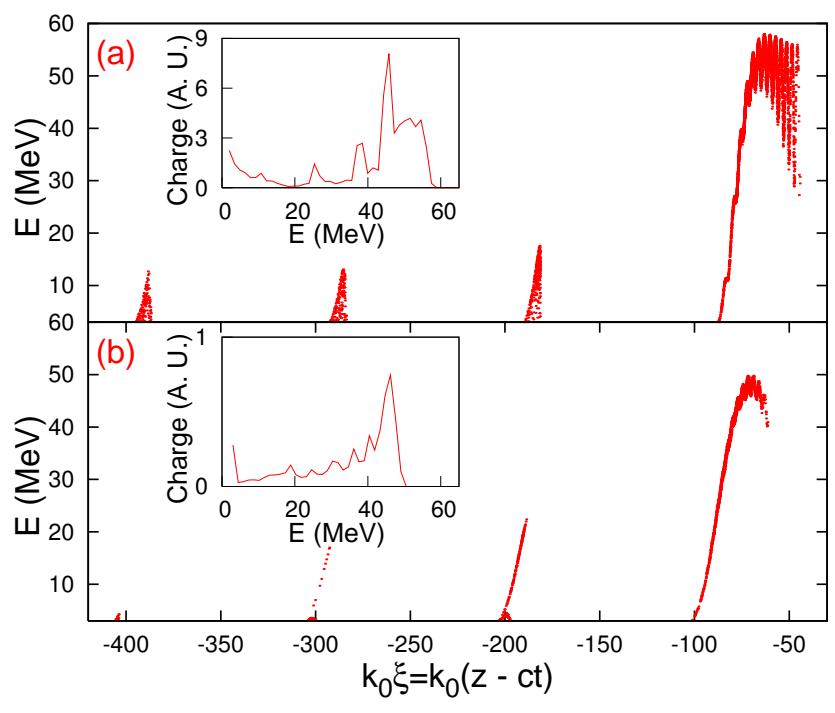

FIG. 3: Energy gain after $300 \mu \mathrm{m}$ as a function of relative phase $\xi=z-c t,(\xi=0$ corresponding to the maximum of the pump pulse). Top : prescribed fields simulation, bottom : PIC simulation.

the plasma wake inhibition reduces the phase space volume of injected particles. This leads to lower trapped charge and to a lesser extent, to a smaller energy spread in the PIC simulation.

Figure 4 shows a more complete comparison of the charge obtained in both simulations (logarithmic scale), with $a_{0}$ varying beetween $a_{0}=0.9$ and $a_{0}=2$. Here, $a_{0}=0.9$ corresponds to the trapping threshold. The hollow squares represent the charge obtained in 1D PIC simulations ; the triangles represent the charge obtained using the prescribed fields simulations. As we can see, the prescribed fields simulation overestimates the charge by a constant factor 8 . The plasma wake inhibition and the reduction of the trapped charge has also been confirmed in 3D hybrid PIC simulations. [17].

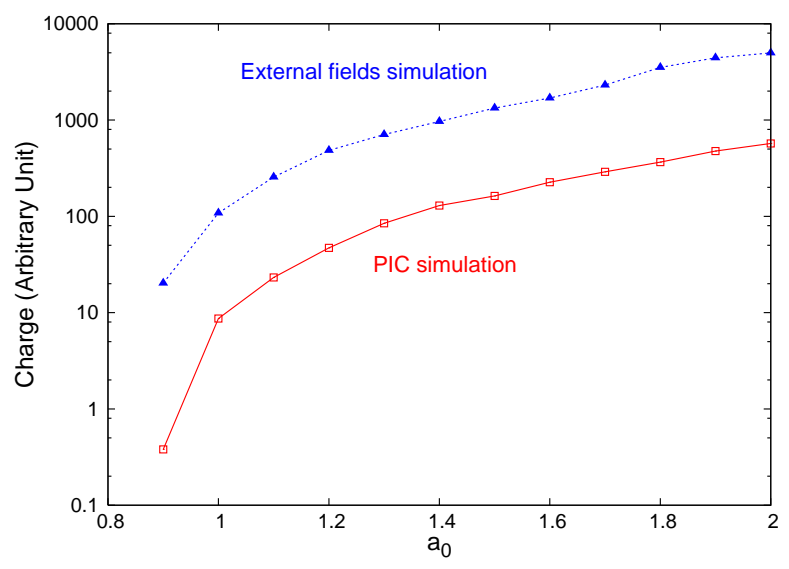

FIG. 4: Comparison of injected charge obtained in PIC simulations and in prescribed fields simulations.

In conclusion, although the previous modelling of the collision of two laser pulses in an underdense plasma gives a good understanding of the general processes at play in the injection of electrons, we have observed and explained an important physical process neglected to date. Using 1D PIC simulations, we have shown that the beatwave does not only preaccelerate electrons but also reduces the wakefield amplitude. In this process, the trapped charge decreases by an order of magnitude and to a lesser extent, the energy spread can be improved. Further studies will focus on minimizing this effect and increasing the injected charge.
[1] E. Esarey, R. Hubbard, W. Leemans, A. Ting, and P. Sprangle, Phys. Rev. Lett. 79, 2682 (1997).

[2] G. Fubiani, E. Esarey, C. Schroeder, and W. Leemans, Phys. Rev. E. 70, 016402 (2004).

[3] T. Tajima and J. M. Dawson, Phys. Rev. Lett. 43, 267 (1979).

[4] A. Pukhov and J. Meyer-ter Vehn, Appl. Phys. B 74, 355 (2002).

[5] S. P. D. Mangles et al., Nature 431, 535 (2004).

[6] C. G. R. Geddes et al., Nature 431, 538 (2004).

[7] J. Faure et al., Nature 431, 541 (2004).

[8] C. DesRosiers, V. Moskvin, A. Bielajew, and L. Papier, Phys. Med. Biol. 45, 1781 (2000).

[9] Y. Glinec et al., Phys. Rev. Letter. 94, 025003 (2005).

[10] D. Umstadter, J.-K. Kim, and E. Dodd, Phys. Rev. Lett.
76, 2073 (1996).

[11] J. Faure, C. Rechatin, A. Norlin, A. Lifschitz, and V. Malka, Nature 444, 737 (2006).

[12] L. Gorbunov and A. Frolov, Plasma Physics Reports 29, 407 (May 2003).

[13] Z.-M. Sheng et al., Phys. Rev. Lett. 88, 055004 (2002).

[14] H. Kotaki, S. Masuda, M. Kando, J. K. Koga, and K. Nakajima, Physics of Plasmas 11, 3296 (2004).

[15] E. Esarey and M. Pilloff, Physics of Plasmas 2, 1432 (1995).

[16] E. Lefebvre et al., Nuclear Fusion 43, 629 (2003).

[17] A. Lifschitz, J.Faure, C. Rechatin, and V. Malka, Submitted to Phys. Rev. Letter. (2007). 